\title{
Mathematical Tables in Reports of the B.A.A.S.
}

For over seventy consecutive years the British Association for the Advancement of Science has had a Committee on Calculation of Mathematical Tables, and we hope to be in a position later to present a detailed account of its membership and activities. Meanwhile we list chronologically the Mathematical Tables published (1877-1929) in the Reports of the B.A.A.S., and a few others related to them, which appeared elsewhere. Practically all of these Tables are by members of the Committee and most of them are in the field of Bessel Functions (1889-1928). There were also some tables in J. W. L. Glaisher's article, "On the law of distribution of prime numbers," 1872 (Transactions, p. 20).

1873 (p. 1-175).-Before any Tables were published there appeared the remarkable 1. Report of the Committee on Mathematical Tables by J. W. L. GLAISHER. This is a sort of catalogue and analysis of Mathematical Tables up to that time. It was an elaboration of the idea illustrated by Augustus De Morgan's article on "Mathematical Tables" in The English Cyclopaedia, Arts and Science Section, v. 7, 1861, cols. 976-1016. Important supplements to Glaisher's Report, by A. Cayley and others, were published in 1875,1878 , 1879, 1880, 1881, and 1883.

1877 (Notices and abstracts, p. 8-14).-1A [added in proof]. J. C. AdAMs, "On the calculation of Bernoulli's numbers up to $B_{62}$ by means of Staudt's theorem," with tables (not published by the Committee) expressing the numbers in (a) vulgar fractions, (b) repeating decimals. The first table was printed also in Jn. f. d. reine u. angew. Math., v. 85, 1878, p. 270-271; and the second is given in Scientific Papers of Adams, v. 1, Cambridge, 1896 , p. $455-458$.

1879 (p. 49-57 + plate I).-2. J. W. L. Glaisher, "Legendrian functions," calculated in 1872. $P_{n}(x)$, for $x=[0.00(0.01) 1 ; 2 \mathrm{D}$ to $18 \mathrm{D}], n=1(1) 7$. A listing of a table by Samuel Houghton for calculation of sun-heat coefficients (p. 66-71) is omitted.

1889 (p. 28-32).-3. A. LodGE, $I_{n}(x)$ for $x=[0.0(0.2) 6.0 ; 12 S], n=0(1) 11$, the last figure approximate. $I_{n}(x)=i^{-n} J_{n}(i x)$. Compare nos. 5, 6.

1893 (p. 82-119).-4. C. E. Bickmore, "Tables connected with the Pellian equation $\left[y^{2}=a x^{2}-1\right]$ from the point where the work was left by Degen in 1817"; report by A. CAYley (p. 73-120) also in Collected Math. Papers, v. 13, 1897, p. 430-467. Extends the table from $a=1001$ to $a=1500$.

1893 (p. 227-279).-5. A. LoDGE, $I_{1}(x)$ for $x=$ [0.001(0.001)5.100; 9D], last figure approximate. With differences. On p. 228 there is a table of $J_{0}(x \sqrt{i})$ for $x=[0.0(0.2) 6.0 ; 9 \mathrm{D}]$. Compare nos. 3, 6 .

1896 (p. 98-149).-6. A. LoDGE, $I_{0}(x)$ for $x=[0.000(0.001) 5.100 ; 9 D]$, last figure approximate. With differences. It was found that nos. 5 and 6 were "subject to errors of two or three units in the last decimal place"; see notes on Table VI, p. xv, of B.A.A.S., Mathematical Tables, v. 6, Bessel Functions, part I, Cambridge, 1937. Compare nos. 3, 5. It was at this time that the Committee recommended the publication of a volume of tables of Bessel functions to $6 \mathrm{D}$. 
1899 (p. 65-120).-7. AliCE LEE, $G(r, \nu)$-integrals, for $\phi=0^{\circ}\left(1^{\circ}\right) 45^{\circ}$, $r=1(1) 50$, where $\tan \phi=\nu / r . G(r, \nu)=\int_{0}^{\pi} \sin ^{r} \theta e^{\nu \theta} d \theta=e^{(1 / 2) \nu \pi} F(r, \nu)$ $=\left[\left(e^{\nu \phi+(1 / 2) \nu \pi}(\cos \phi)^{r+1} / \sqrt{r-1}\right)\right] H(r, \nu)$. The tables are to $7 \mathrm{D}$, with first and second differences, of $\log F(r, \nu)$ and $\log H(r, \nu)$. These are in a report of Karl Pearson, and not of the Mathematical Tables Committee. They are reprinted in Table LIV (p. lxxxi-lxxxiii, 126-142) of Tables for Statisticians and Biometricians, ed. K. Pearson, part I, London, 1914; second ed., 1924; third ed. 1930.

1907 (p. 94-97 + folding plate).-8. A. LoDGE, Table I: $\log R_{n}$ to $8 \mathrm{D}$; Table II: $\log \left[R_{n}(2 / \pi)^{1 / 2}\right]$ to $7 \mathrm{D}$; Table III: $Q_{0}(x) ; 10 \mathrm{D}$, $\log \left[-Q_{0}(x)\right] ; 8 \mathrm{D}, \log \left(-\sin \alpha_{0}\right) ; 8 \mathrm{D},-\sin \alpha_{0} ; 9 \mathrm{D}, \log \left(-x \sin \alpha_{0}\right) ; 8 \mathrm{D}$, all for $x=10(10) 100(100) 1000$, and $n=0(1 / 2)(13 / 2)$, where $J_{n}(x)=$ $(2 / \pi x)^{1 / 2} R_{n}(x) \cos \left(x+\alpha-\frac{\pi}{4}-n \frac{\pi}{4}\right), R_{n}{ }^{2}=P_{n}{ }^{2}+Q_{n}{ }^{2}, \sin \alpha=Q_{n} / R_{n}$, $P_{n}(x)=1-\frac{\left(4 n^{2}-1\right)\left(4 n^{2}-3^{2}\right)}{1 \cdot 2(8 x)^{2}}+\frac{\left(4 n^{2}-1\right) \cdots\left(4 n^{2}-7^{2}\right)}{1 \cdot 2 \cdot 3 \cdot 4(8 x)^{4}}-\cdots ; Q_{n}(x)=$ $\frac{4 n^{2}-1}{8 x}-\frac{\left(4 n^{2}-1\right)\left(4 n^{2}-3^{2}\right)\left(4 n^{2}-5^{2}\right)}{1 \cdot 2 \cdot 3(8 x)^{3}}+\cdots$. Concluded in no. 9.

1909 (p. 33-36).-9. A. Lodge, Table IV: $Q_{i}(x), i=0,1, \cdots, 6 ; x=$ [10(10) $100(1000 ; 9 \mathrm{D}-10 \mathrm{D}]$. Table V: $\log \left[8 x a /\left(4 n^{2}-1\right)\right], n=0(1 / 2)(13 / 2)$; $x=[10(10) 100(100) 1000 ; 7 D]$, "last figure approximative." Varying values for $a$. Continued from no. 8.

1911 (p. 67-71).-10. G. Greenhill, Specimen tables of the elliptic function for modular angle $\theta=45^{\circ}$ and $\theta=75^{\circ}$, the argument proceeding by degrees of the quadrant of the quarter period $K$ or $F\left(90^{\circ}\right)$.

1911 (p. 73-78).-11. J. R. AIREY, Neumann functions $G_{n}(x)$ and $Y_{n}(x)$, i.e., $G_{0}(x), G_{1}(x), Y_{0}(x), Y_{1}(x)$, for $x=[0.0(0.1) 16.0 ; 7 \mathrm{D}]$, the greatest error being $10^{-7}$. There is a reference to W. S. Aldis, R. So. London, Proc., v. 66, 1900 , p. 41 , for a table of $G_{0}(x)$ and $G_{1}(x)$ for $x=[0.0(0.1) 6.0 ; 21 \mathrm{D}]$. Compare nos. $15,16$.

1912 (p. 39-55).-12. G. Greenhill, Four specimal tables of elliptic integrals, calculated for the modular angles $\theta=15^{\circ}, K^{\prime}=K \sqrt{3} ; 45^{\circ}$, $K^{\prime}=K ; 75^{\circ}$; and $80^{\circ} 1, K=2 K^{\prime}$.

1912 (p. 56-68).-13. A. G. WeBsTER, Ber $x$, bei $x$, and their derivatives, $x=[0.0(0.1) 10.0 ; 9 \mathrm{D}]$, with 7 differences. "The last 35 entries for the derivative of bei $(x)$ have been corrected to 8D." (Committee's report, 1929). Published because of "especial service to electrical engineering." $\mathrm{H}$. G. SAVIDGE in no. 25 noted that there was an error in Webster's table of "bei $x$ which necessitated the recalculation of part of the table. The error became considerable as the argument increases, and the corrected figures" from $x=6.5$ to 10.5 are given 1916 , p. 122 . These are the 41 , not " 35 items."

1913 (p. 88-113 + 4 plates).-14. G. GreENHILL, Elliptic functions, for which the ratio of the periods $K / K^{\prime}=1 / \sqrt{2}, \sqrt{2}, 2 \sqrt{2}, 2 \sqrt{3}, 3,3 / \sqrt{2}, 3 \sqrt{2}$ $3 \sqrt{3}, 4,5$; various graphs including those corresponding to these ratios.

1913 (p. 115-130).-15. J. R. AIREy, Neumann functions $G_{0}(x)$ and $G_{1}(x)$, or Bessel functions of the second kind, for $x=[0.00(0.01) 16.00 ; 7 \mathrm{D}]$. Compare nos. 11, 16. 
1914 (p. 75-82).-16. J. R. Airey, Neumann functions, $Y_{0}(x), Y_{1}(x)$, for $x=[0.02(0.02) 15.50 ; 6 \mathrm{D}]$, correct to $6 \mathrm{~S}$. Compare nos. 11, 17.

1914 (p. 83-86).-17. J. R. AIREy, $G_{n}(x)$ for $x=[0.0(0.1) 6.0(0.5) 16.0$; $5 \mathrm{D}]$, and $n$ ranging irregularly for 3 to 14 values. Compare nos. 16, 19.

1914 (p. 87-102).-18. A. T. Doodson, Bessel functions of half integral order, $S_{n}(x)=(\pi x / 2)^{1 / 2} J_{n+1 / 2}(x), C_{n}(x)=(-1)^{n}(\pi x / 2)^{1 / 2} J_{-n-1 / 2}(x), S_{n}{ }^{\prime} \equiv$ $d S_{n}(x) / d x, C_{n}{ }^{\prime}(x) \equiv d C_{n}(x) d x / d x,{S_{n}}^{2}+C_{n}{ }^{2},{S_{n}}^{\prime 2}+C_{n}{ }^{2}$, and their common logarithms, for $x=[1(1) 10 ; 7 \mathrm{~S}]$, and $n$ ranging irregularly for 5 to 24 values. Compare no. 24.

1915 (p. 27-36).-19. J. R. AIrey, Table I: $J_{n}(x)$, of integral order, for $x=[0.2(0.2) 6.0(0.5) 16.0 ; 6 \mathrm{D}$ to $10 \mathrm{D}]$, varying values of $n$. Table II : $G_{0}(x)$, for $x=[6.5(0.5) 15.5 ; 10 \mathrm{D}] ; Y_{0}(x), Y_{1}(x)$ for $x=[0.1(0.1) 6.0(0.5) 15.5 ; 10 \mathrm{D}]$. Table III : $Y_{n}(x)$ for $x=[0.2(0.2) 8.0(0.5) 15.5 ; 6 \mathrm{D}]$, for varying $n$. Compare no. 11.

1915 (p. 36-38).-20. H. G. SAvidGe, Ker, kei, and their first derivatives, for $x=[0.0(0.1) 10.0 ; 7 \mathrm{D}-9 \mathrm{D}]$.

1916 (p. 59-89).-21. J. R. AIrey, Table I: Sines and cosines for $x=$ $\left[0^{r} .000\left(0^{r} .001\right) 1^{r} .600 ; 11 D\right]$; Table II : Table of $x-\sin x$ and $1-\cos x$, with first differences, for $x=\left[0^{r} .00001\left(0^{r} .00001\right) 0^{r} .00100 ; 11 \mathrm{D}\right]$. Compare no. 22.

1916 (p. 90-91).-22. A. T. Doodson, Table III: $\operatorname{Sin} x, \cos x$, for $x=$ $[0.0(0.1) 10.0 ; 15 \mathrm{D}]$. Second editions of nos. 21 and 22 appeared in B.A.A.S., Mathematical Tables, v. 1, London, 1931, p. 8-23, 3.

1916 (p. 92-96).-23. J. R. AIrEy, Bessel and Neumann functions of equal order and argument, $J_{n}(x), J_{n-1}(x), G_{n}(x), G_{n-1}(x),-Y_{n}(x),-Y_{n-1}(x)$, for $n=[1(1) 50(5) 100(10) 200(20) 400(50) 1000(100) 2000(500) 5000(1000)$ 20000(5000)30000(10000)50000, 100000, 500000, 1000000; 6D].

1916 (p. 97-107).-24. A. T. Doodson, $S_{n}, C_{n}, S_{n}{ }^{\prime}, C_{n}{ }^{\prime}, S_{n}{ }^{2}+C_{n}{ }^{2}$, $S_{n}{ }^{2}+C_{n}{ }^{2}$ and their logarithms for $x=[1.1(0.1) 1.9 ; 7 \mathrm{~S}]$ and for 11 different values of $n$. Compare nos. 18, 30.

1916 (p. 108-122).-25. H. G. SAvidge, (1) $X b(x)=\operatorname{ber}^{2} x+\operatorname{bei}^{2} x$; (2) $V b(x)=\operatorname{ber}^{\prime 2} x+\operatorname{bei}^{\prime 2} x ;$ (3) $Z b(x)=$ ber $x \operatorname{ber}^{\prime} x+$ bei $x$ bei $^{\prime} x$; (4) $W b x=$ ber $x$ bei $^{\prime} x$ - bei $x \operatorname{ber}^{\prime} x$; (5)-(8) $X k(x)=\operatorname{ker}^{2} x+\operatorname{kei}^{2} x, V k(x)$, $-Z k(x),-W k(x)$; (9) $\operatorname{Vr}(x)=\operatorname{ber}^{\prime} x \operatorname{ker}^{\prime} x+\operatorname{bei}^{\prime} x \operatorname{kei}^{\prime} x$. (10) $V u(x)=$

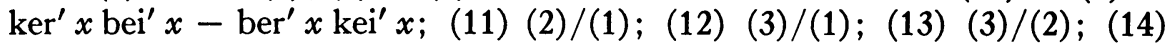
$(4) /(2)$. All of these functions are for the range $x=[0.0(0.2) 10.0 ; 5 S-7 S]$. See no. 13.

1916 (p. 123-126).-26. G. N. WAtson, (a) $10+\ln \Gamma(1+x)$, for $x=[.005(0.005) 1.000 ; 10 \mathrm{D}]$; (b) $10+\int_{0}^{x} \log \Gamma(1+t) d t$, for $x=[0.1(0.1)-$ $1.00 ; 10 \mathrm{D}]$; (c) $d \ln \Gamma(x) / d x$, for $x=[1(1) 101 ; 13 \mathrm{D}]$ and $x=\left[1 \frac{1}{2}(1) 100 \frac{1}{2}\right.$; 13D].

1919 (p. 43-77).-27. R. L. HippisLey, Tables I-III of the lemniscate function, $K=K^{\prime}$, and $K=2 K^{\prime}, K=4 K^{\prime}$, for $r=[0(1) 45 ; 10 \mathrm{D}]$. "Some fault was found with the final decimals in the table of the lemniscate functions $K=K^{\prime}$, to $7 \mathrm{D}, \theta=45^{\prime}, 1912$, p. 50 . As this table has been used as the base of the calculation of the table for $K=(2,3,4,5,7 \cdots) K^{\prime}$, by means of a transformation of the order $2,3,4, \cdots$ as explained 1913, p. 88, it was decided to make a recalculation to a higher degree of accuracy, going to 10 decimals." Compare no. 12. 
1919 (p. 78-79.-28. Table IV. $e^{-n \pi / 16}$ for 60 integral values of $n$ from $n=1$ to $n=242$, to $20 \mathrm{D}$.

1919 (p. 80-81).-29. A. Lodge, Table V. Lemniscate seven-section, to 15D; Table VI. Lemniscate seventeen-section to $15 \mathrm{D}$.

1922 (p. 263-270).-30. A. T. Doodson, Riccati-Bessel functions $S_{n}, C_{n}$, $S_{n}{ }^{\prime}, C_{n}{ }^{\prime}, S_{n}{ }^{2}+C_{n}{ }^{2}, S_{n}{ }^{2}+C_{n}{ }^{\prime 2}$, and their logarithms, for $x=[0.1(0.1) 0.9$; $7 \mathrm{~S}$ or $8 \mathrm{~S}$ ], and 8 different values of $n$. Compare no. 18.

1922 (p. 271-272).-31. J. R. AIREy, Zeros of Bessel functions $J_{n}(x)$ of high order, first 10 zeros, for $n=[0(1) 10(5) 20(10) 50,75,100(100) 500,750$, $1000 ; 5 \mathrm{D}$ or $6 \mathrm{D}]$.

1923 (p. 287-289).-32. J. R. AIREY, $\sin x$, and $\cos x, x=\left[0^{r}\left(1^{r}\right) 100\right.$; 15D]. Compare nos. 21, 22, 35.

1923 (p. 290-293).-33. J. R. AIREy, (a) $J_{\nu}(\nu), J_{\nu-1}(\nu),-Y_{\nu}(\nu)$, $-Y_{\nu-1}(\nu), \nu=[0(1 / 4) 10 ; 6 \mathrm{D}]$; (b) When $\nu$ is not an integer $J_{\nu}(x)=$ $\frac{1}{\pi} \int_{0}^{\pi} \cos (\nu \theta-x \sin \theta) d \theta-\sin \frac{\nu \pi}{\pi} \int_{0}^{\infty} e^{-x} \sinh \theta e^{-\nu \theta} d \theta$, as shown by Schläfli. Let $F_{\nu}(\nu)$ denote the last term. $F_{\nu}(\nu), F_{\nu-1}(\nu), F_{-\nu}(\nu), F_{-\nu+1}(\nu)$, for $\nu=$ [0(1/4) 10;6D]; (c) Lommel-Weber $\Omega$ function, defined by $\Omega_{\nu}(x)=$ $\frac{1}{\pi} \int_{0}^{\pi} \sin (x \sin \phi-\nu \phi) d \phi$, is tabulated for $\nu=[0(1 / 4) 10 ; 6 \mathrm{D}]$. This definition of $\Omega_{\nu}(x)$ is that of Airey's table no. 36, of Nielsen (1904), and of Jahnke and Emde (1938). Airey here erred in defining $\Omega_{\nu}(x)$ as the negative of this value.

1923 (p. 293-298).-34. A. E. KenNelly, Bessel functions of negative semi-imaginary argument, $\left.z \sqrt{-i}, J_{0}\left(z \overline{45^{\circ}}\right), J_{1} \sqrt{45^{\circ}}\right)$, for $z=[0(0.1) 10 ; 5 \mathrm{D}$ or $6 \mathrm{D}]$. Also their application to determination of the alternating skin-effect impedance ratio, $Z^{\prime} / R$, of straight uniform round copper wires, remote from other active conductors, according to the formula $Z^{\prime} / R=\left[\left(z \backslash \overline{45^{\circ}}\right) / 2\right]$ $\times J_{0}\left(z \overline{45^{\circ}}\right) / J_{1}\left(z \overline{45^{\circ}}\right)$, for $z=[0.0(0.1) 10.0 ; 6 \mathrm{D}]$.

1924 (p. 275-278).-35. J. R. AIREY, Sin $x$, and $\cos x$, for $x=[10.0(0.1)$ $20.0(0.5) 50.0 ; 15 D]$. Reprinted in B.A.A.S., Mathematical Tables, v. 1, London, 1931, as part of p. 4-7. Compare nos. 32, 47.

1924 (p. 279-287).-36. J. R. AIREy, Lommel-Weber functions of zero and unit orders, $\Omega_{0}(x)$ and $\Omega_{1}(x)$ for $x=[0.00(0.02) 16.00 ; 6 \mathrm{D}]$. Compare nos. 33, 39.

1924 (p. 287-295).-37. J. R. Arrey, Bessel-Clifford functions of zero and unit orders, $C_{0}(x)$ and $C_{1}(x)$ for $x=[0.00(0.02) 20.00 ; 6 \mathrm{D}]$.

1925 (p. 221-233).-38. J. R. AIREy, Bessel functions of half odd integral order, $J_{\nu}(x)$, to $12 \mathrm{D}$; for (according to A. N. Lowan)

$\begin{array}{lllllccc}x & \nu & x & \nu & x & \nu & x & \nu \\ 1 & \frac{-1(2) 23}{2} & 6 & \frac{-15(2) 47}{2} & 11 & \frac{-27(2) 63}{2} & 16 & \frac{-37(2) 79}{2} . \\ 2 & \frac{-5(2) 29}{2} & 7 & \frac{-17(2) 51}{2} & 12 & \frac{-29(2) 67}{2} & 17 & \frac{-39(2) 81}{2} \\ 3 & \frac{-7(2) 35}{2} & 8 & \frac{-19(2) 53}{2} & 13 & \frac{-31(2) 69}{2} & 18 & \frac{-41(2) 85}{2} \\ 4 & \frac{-9(2) 39}{2} & 9 & \frac{-21(2) 57}{2} & 14 & \frac{-33(2) 73}{2} & 19 & \frac{-43(2) 87}{2} \\ 5 & \frac{-11(2) 43}{2} & 10 & \frac{-23(2) 61}{2} & 15 & \frac{-35(2) 75}{2} & 20 & \frac{-45(2) 89}{2}\end{array}$


1925 (p. 234-253).-39. J. R. Arrey, $J_{1 / 2}(x), J_{-1 / 2}(x), \Omega_{1 / 2}(x), \Omega_{-1 / 2}(x)$, for $x=[0(0.02) 20.00 ; 6 \mathrm{D}]$. Compare no. 36 .

1926 (p. 273-275).-40. J. R. AIREy, Fresnel integrals, $\mathrm{S}(x)$ and $\mathrm{C}(x)$, for $x=[0.0(0.1) 20.0 ; 6 \mathrm{D}]$.

1926 (p. 276-294).-41. J. R. ArRey, Confluent hypergeometric function $M(\alpha \cdot \gamma \cdot x)$, for 31 values of $x=0.0(0.1) 1.0(0.2) 3.0(0.5) 8.0, \gamma= \pm 1 / 2$, $\pm 3 / 2, \alpha=[-4(1 / 2) 4 ; 6 \mathrm{D}]$. Compare no. 43.

1926 (p. 295-296).-42. J. R. AIREY, $\sinh x, \cosh x$, for $x=[0.1(0.1) 10.0$; 15D]. Reprinted in B.A.A.S., Mathematical Tables, v. 1, London, 1931, p. 30. Compare $47(\mathrm{c}), 52$.

1927 (p. 220-244).-43. J. R. AIREY, Confluent hypergeometric function $M(\alpha \cdot \gamma \cdot x), \gamma=1,2,3,4, \alpha=-4(0.5) 4, x=[0.00(0.02) 0.10(0.05) 1.0-$ (0.1) $2.0(0.2) 3.0(0.5) 8.0 ; 6 \mathrm{D}$ or $6 \mathrm{~S}]$; and further values of the function for $\gamma= \pm 1 / 2, \pm 3 / 2$. Compare no. 41,55 .

1927 (p. 245-251).-44. J. R. AIREy, Exponential, sine, and cosine integrals: (a) $E i( \pm x), x=[5.0(0.1) 15.0 ; 5 \mathrm{D}$ to $14 \mathrm{D}]$; (b) $\mathrm{Si}(x)$ and $\mathrm{Ci}(x)$, for $x=[5.0(0.1) 20.0 ; 10 \mathrm{D}]$. Compare no. 48 .

1927 (p. 252-253).-45. J. R. AIrey, Zeros of Bessel functions $J_{\nu}(x)$ of small fractional order, the first zero $\rho_{1}$, to $6 \mathrm{~S}$, for $\nu=-1.00(0.01) 1.00$.

1927 (p. 253-254).-46. J. R. AIREy, First 10 zeros, to 5D, of ber $x$, bei $x$, ker $x$, kei $x, \operatorname{ber}^{\prime} x$, bei $^{\prime} x, \operatorname{ker}^{\prime} x, \operatorname{kei}^{\prime} x$.

1928 (p. 305-316). -47. J. R. AIREY, (a) $\sin x$ and $\cos x$, for $x=[20.0-$ (0.2) 40.0;15D]; compare 32; (b) $e^{\pi x}$, for $x=[-4.0(0.5) 4.0 ; 20 \mathrm{D}]$, and (c) $\sinh \pi x, \cosh \pi x$, for $x=[0.01(0.01) 4.00 ; 15 D]$. In B.A.A.S., Mathematical Tables, v. 1, Cambridge, 1931, p. 5-6, 26-29, (a) and (c) were reprinted.

1928 (p. 316-323). -48. J. R. Airey, (a) $\mathrm{Si}(x)$ and $\mathrm{Ci}(x)$, for $x=$ [20.0(0.2)40.0;10D]; compare no. 44; (b) Derivatives of Bessel functions $\frac{\partial}{\partial \nu} J_{\nu}(x), x=[0.0(0.1) 20.0 ; 6 \mathrm{D}], \nu= \pm 1 / 2, \pm 3 / 2$. Such derivatives are closely related to sine and cosine integrals. For nos. 44, and 48 (a) see B.A.A.S., Mathematical Tables, v. 1, Cambridge, 1931, p. xv, etc.

1928 (p. 324-340). -49. J. R. AIrEy, The probability integral $I_{0}=$ $\int_{x}^{\infty} e^{-(1 / 2) t^{2}} d t$ and its integrals. Let $I_{n}=\int_{x}^{\infty} I_{n-1}(x) d x$. The tables are for $I_{n}(x)$, for $n=0,1, \cdots 20, x=[0.0(0.1) 6.6 ; 10 \mathrm{D}]$; and for $I_{n}(-x), n=0,1,2$, .. 21, for $x=[0.0(0.1) 10.0 ; 10 \mathrm{D}]$.

1929 (p. 251-262). -50. A. LoDGe, Harmonic series $\phi(x)$, for $x=$ $[0.0(0.1) 60.3 ; 16 \mathrm{D}]$ and $x=[50.00(0.01) 51.00 ; 10 \mathrm{D}] . \phi(x)=\gamma+\frac{d}{d x} \ln \Gamma(1+x)$, where $\gamma$ is Euler's constant. This is the last table published in the B.A.A.S. Reports.

In its report of 1929 the Committee stated that the "following Tables from other sources are intended to supplement those which have appeared in the Committee's reports" to the B.A.A.S.:

51. J. W. L. Glaisher, "Tables of the numerical values of the sineintegral, the cosine-integral, and exponential-integral," R. So. London, Trans., v. 160,1870 , p. $367-388$. Tables I-IX: $\operatorname{Si}(x), \operatorname{Ci}(x), \operatorname{Ei}( \pm x)$, for $x=[0(0.01) 1 ; 18 \mathrm{D}],[1(0.1) 5(1) 15 ; 11 \mathrm{D}]$, with differences to the third order up to $x=5$; Table $\mathrm{X}$ : $\mathrm{Si}(x), \mathrm{Ci}(x)$, for $x=[20(5) 100(10) 200(100) 1000$ - 
(1000)11000; 7D], $x=\left[10^{5}, 10^{6}, 10^{7}, 10^{8}, \infty ; 7 \mathrm{D}\right]$. Tables XI, XII : maxima and minima of $\mathrm{Si}(x)$ and $\mathrm{Ci}(x)$, to 7D. The "Table of Constants" (p. 370) gives the values of $x \Gamma(x+1)$ and its $\ln$, for $x=[2(1) 71 ; 20 \mathrm{~S}$, and 10D respectively].

52. D. F. E. MeIssel, Tafel der Bessel'schen Functionen $I_{k}{ }^{0}$ und $I_{k}{ }^{1}$ von $k=0$ bis $k=15.5$ berechnet," Preuss. Akad. Wissen., Abh. 1888, Berlin, 1889,23 p. $I_{k}{ }^{0}, I_{k}{ }^{1}$ are simply $J_{0}(x), J_{1}(x)$. The first 10 zeros of $J_{0}(x)$ are given on page 3 and the values of $J_{0}(x), J_{1}(x)$ are given (p. 4-23), for $x=[0.00(0.01) 15.50 ; 15 \mathrm{D}]$. Reprinted as Table I, in A. GRAY and G. B. Mathews, $A$ Treatise on Bessel Functions, second ed. by A. Gray and T. M. Macrobert, London, 1922, p. 267-286. A correction of $J_{0}(1.71)$, suggested by Meissel, is here made, but there were three other errors in Meissel

$\begin{array}{lllll}J_{0}(0.62), & \text { for } & 0.90518, & \text { read } & 0.90618 \\ J_{0}(1.89), & \text { for } & 0.28663, & \text { read } & 0.28763 \\ J_{1}(7.87), & \text { for } & 0.21401, & \text { read } & 0.21407\end{array}$

The first and third of these, as well as $J_{0}(3.07)$, which is correct in Meissel, are incorrect in Gray and Mathews 2. The second is incorrect in G. and M.1. Table II (p. 286-299), of Gray and Mathews, is from an unpublished ms. of Meissel of $J_{n}(x)$ for $x=0(1) 24, n=[0(1) 60 ; 16 \mathrm{D}]$. In this table Airey (no. 42, p. 297) noted the following errors: $J_{4}(5)$ for 26304 , read 23604; $J_{23}(6)$ for 02496 , read $02495 ; J_{30}(14)$ for 538 , read $534 ; J_{31}(16)$ for 49322 , read 94322 . Another error in G. and M.1 is $J_{0}(5.90)$, for 0.11203 , read 0.12203 . Table IV of Gray and Mathews is taken from D. F. E. Meissel, "Ueber die Bessel'schen Functionen $J_{k}^{0}$ und $J_{k}{ }^{1}$." Progr., Kiel, 1890, and contains the first 50 roots of $J_{1}(x)=0$, with the corresponding maximum or minimum values of $J_{0}(x)$.

53. R. W. Willson and B. O. PeirCE, "Table of the first forty roots of the Bessel equation $J_{0}(x)=0$ with the corresponding values of $J_{1}(x)$," Amer. Math. So., Bull., v. 3, 1896, p. 153-155. The value of $J_{1}(x)$ for the 35th zero is incorrect; for 0.07635913 , read 0.07636383 . Reprinted with the error in Gray and Mathews.

54. H. E. WriNCH and D. M. Wrinch, "Tables of Bessel functions," Phil. Mag., s. 6, v. 47, 1924, p. 62-65, $I_{n}(x)$, for large integral values of $x$ from 16 to 37 with tables of $J_{2}(x) / I_{2}(x)$, and $J_{2}(x) / I_{3}(x)$, for $x$ from 1 to 15 .

55. H. E. WRINCH and D. M. WRINCH, "Roots of hypergeometric functions with a numerator and four denominators," Phil. Mag., s. 7, v. 1, 1926, p. 273-276. First 9 roots for various values of the five parameters. Compare no. 41.

56. W. S. Aldis, "Tables for the solution of the equation $\frac{d^{2} y}{d x^{2}}+\frac{1}{x} \frac{d y}{d x}$ $-\left(1+\frac{y^{2}}{x^{2}}\right) y=0$," R. So. London, Proc., v. 64, 1899, p. 203-223. $I_{0}(x)$, $I_{1}(x)$, for $x=[0.1(0.1) 6.0(1) 11 ; 18 \mathrm{D}$ to $21 \mathrm{D}] ; K_{0}(x), K_{1}(x)$, for $x=$ [0.1(0.1)120;9D to $21 \mathrm{D}]$.

57. A. LodGe, Zonal harmonics, $P_{n}(\cos \theta), \theta=0^{\circ}\left(5^{\circ}\right) 90^{\circ}, n=[1(1) 20$; 7D], R. So. London, Trans., v. 203 A, 1904, p. 100-101; "llast figure approximate." Appendix to a paper, "On the acoustic shadow of a sphere," by Rayleigh. Compare RMT 92. 
58. J. C. Adams, "Useful formulae, connecting Legendre's coefficients, which are employed in the theory of terrestrial magnetism," Scientific Papers of Adams, v. 2, Cambridge, 1900, p. 243-296. On p. 268-281, Gaussian functions $G_{n}{ }^{m}(\mu), n=0(1) 10, m=0(1) 10, \mu=[0.00(0.05) 1 ; 10 \mathrm{D}]$.

\section{Authors}

AdAMS, 1A, 58

AIREY, 11, 15, 16, 17, 19, 21, 23, 31, 32, 33, $35-49,52$

ALDIS, 11, 56

BICKMORE, 4

CAYLEy, 4

Doodson, 18, 22, 24, 30

Glaisher, 1, 2, 51, 1872

Gray and Mathews, 52, 53

Greenhill, 10, 12, 14

HipPisley, 27
KenNelly, 34

LEE, 7

Lodge, 3, 5, 6, 8, 9, 29, 50, 57

MeISSEL, 52

PeIRCE, 53

SAVIDGE, 20, 25

WATSON, 26

WEBSTER, 13

WILLSON, 53

Wrinch, 54, 55

Anonymous, 28

R. C. A.

\section{RECENT MATHEMATICAL TABLES}

98[S].-Project for Computation of Mathematical Tables (A. N. Lowan, technical director), Miscellaneous Physical Tables. Planck's Radiation Functions, and Electronic Functions. Prepared by the Federal Works Agency, Works Projects Administration for the City of New York, conducted under the sponsorship of the National Bureau of Standards. New York, 1941, vii, 58 p. $20.2 \times 26.3 \mathrm{~cm}$. Reproduced by a photo offset process. Sold by the U. S. Bureau of Standards, Washington, D. C. The work was not distributed until 1943. $\$ 1.50$; foreign price $\$ 1.75$.

The tables of Radiation Functions are reprinted from Optical So. Amer., Jn., Feb. 1940. A body of alsolute temperature $T$ emits electromagnetic waves of all possible wave lengths $\lambda$. The radiated energy is however very unequally distributed among the waves of different length. The radiation and photon functions are defined as

$R_{\lambda}=c_{1} \lambda^{-5}\left(e^{c_{2} / \lambda T}-1\right)^{-1} ; R_{0-\lambda}=\int_{0}^{\lambda} R_{\lambda} d \lambda ; N_{\lambda}=2 \pi c \lambda^{-4}\left(e^{c_{2} / \lambda T}-1\right)^{-1} ; N_{0-\lambda}=\int_{0}^{\lambda} N_{\lambda} d \lambda$.

In a region of wave lengths ranging from zero to $\lambda$ the integrals $R_{0-\lambda}$ and $N_{0-\lambda}$ express, respectively, the rates of emission of energy and photons per units of area and time. The letters $c, c_{1}, c_{2}$ have special numerical values. It is chiefly in the values of these constants that there is a difference between the various tables previously published and listed on p. $12-13 ; c$ represents the velocity of light $\left(\mathrm{cm} . / \mathrm{sec}\right.$.). The values of $c_{1}$ and $c_{2}$ which were adopted after consultation with L. J. Briggs and H. T. Wensel of the National Bureau of Standards use numbers 3.732 and 1.436 while the recent tables of P. Moon ${ }^{1}$ use 3.697 and 1.432 respectively. On p. 4 of the introduction a way of correcting for a change in $c_{1}$ and $c_{2}$ is explained. Remarks are made also on the number of significant figures in the tabulated entries and on the method of interpolation for $\lambda$. In making the computations results obtained by means of series were checked by evaluating the integrals by a method of numerical integration. In Table I we are given values of $R_{0-\lambda} / R_{0-\infty}, R_{\lambda} / R_{\lambda \max }, N_{0-\lambda} / N_{0-\infty}$, $N_{\lambda} / N_{\lambda_{\max }}$, for $\lambda T=0.050(0.001) \cdot 1(0.005) .4(0.01) .6(.2) 1(0.05) 2$. In Table II are values of $R_{0-\lambda}, R_{\lambda}, N_{0-\lambda}, N_{\lambda}$, for $\lambda$ (in microns) $=.5(0.01) 1(0.05) 4(0.1) 6(0.2) 10(0.5) 20$. In Table III are given values of $N_{\lambda}$, to $4 \mathrm{D}$, for $T=1000^{\circ} \mathrm{K}, 1500^{\circ} \mathrm{K}, 2000^{\circ} \mathrm{K}, 2500^{\circ} \mathrm{K}, 3000^{\circ} \mathrm{K}, 3500^{\circ} \mathrm{K}$, $6000^{\circ} \mathrm{K}$, and $\lambda=0.25(0.05) 1.6(0.2) 3(1) 10$. The last table is to be used for changes in $c_{1}$ and $c_{2}$, from $4 \mathrm{D}$ to $5 \mathrm{D}$ are given for $\lambda T=0.025(.005) .13(.01) .2(.05) .6(.1) 1(.5) 2$. 